\title{
A Gold Standard, CRISPR/Cas9-Based Complementation Strategy Reliant on 24 Nucleotide Bookmark Sequences
}

\author{
François M. Seys ${ }^{1}$, Peter Rowe ${ }^{1,+}{ }^{\text {, Edward L. Bolt }}{ }^{2}{ }^{\mathbb{D}}$, Christopher M. Humphreys $^{1}$ and \\ Nigel P. Minton $1,3, *$ (D) \\ 1 Clostridia Research Group, BBSRC/EPSRC Synthetic Biology Research Centre (SBRC), \\ School of Life Sciences, Biodiscovery Institute, University of Nottingham, Nottingham NG7 2RD, UK; \\ francois.seys@nottingham.ac.uk (F.M.S.); pete@deepbranchbio.com (P.R.); \\ c.humphreys@nottingham.ac.uk (C.M.H.) \\ 2 School of Life Sciences, Queen's Medical Centre, Nottingham NG7 2UH, UK; ed.bolt@nottingham.ac.uk \\ 3 NIHR Nottingham Biomedical Research Centre, Nottingham University Hospitals NHS Trust, \\ Nottingham NG7 2RD, UK \\ * Correspondence: nigel.minton@nottingham.ac.uk; Tel.: +44-(0)115-846-7458 \\ + Present address: Deep Branch Biotechnology, Nottingham NG8 1AA, UK.
}

Received: 3 March 2020; Accepted: 20 April 2020; Published: 23 April 2020

\begin{abstract}
Phenotypic complementation of gene knockouts is an essential step in establishing function. Here, we describe a simple strategy for 'gold standard' complementation in which the mutant allele is replaced in situ with a wild type (WT) allele in a procedure that exploits CRISPR/Cas9. The method relies on the prior incorporation of a unique 24 nucleotide (nt) 'bookmark' sequence into the mutant allele to act as a guide RNA target during its Cas9-mediated replacement with the WT allele. The bookmark comprises a 23 nt Cas 9 target sequence plus an additional nt to ensure the deletion is in-frame. Here, bookmarks are tailored to Streptococcus pyogenes CRISPR/Cas9 but could be designed for any CRISPR/Cas system. For proof of concept, nine bookmarks were tested in Clostridium autoethanogenum. Complementation efficiencies reached 91\%. As complemented strains are indistinguishable from their progenitors, concerns over contamination may be satisfied by the incorporation of 'watermark' sequences into the complementing genes.
\end{abstract}

Keywords: bookmark; CRISPR/Cas9; complementation; Clostridium; knock-out

\section{Introduction}

The genus Clostridium encompasses many species of medical and industrial interest, including Clostridium difficile [1], Clostridium botulinum [2], Clostridium acetobutylicum [3], and Clostridium autoethanogenum [4]. Accordingly, substantial efforts have been expended in recent years to develop convenient and standardised genetic toolboxes to enable the systematic study and engineering of clostridia [5-8]. A fundamental requirement has been the ability to establish gene function through precise gene knock-out $(\mathrm{KO})$ and the subsequent phenotypic comparison of the mutant $\mathrm{KO}$ and progenitor strain, typically, the wild type (WT). However, before a particular change in phenotype may be definitively assigned to the absence of the disrupted gene, it is essential to carry out a complementation study to demonstrate that the introduction of a functional copy of the deleted gene restores the phenotype of the KO strain to that of the progenitor [9]. This rules out the possibility that other, ancillary mutations are responsible for the observed phenotype rather than inactivation of the targeted gene. However, the complementation method itself can also cause unintended changes in phenotype, particularly if the introduced gene is located on an autonomous, multicopy plasmid. 
To circumvent this, we have previously reported the use of the pyrE locus as a genomic site at which single copies of the gene may be easily inserted $[8,10]$. By necessity, however, this strategy requires the prior construction of a specific pyrE mutant and assumes that the positioning of the gene at this location has no unforeseen effects.

A 'gold standard' complementation would ideally involve the restoration of the deleted allele to the WT at its original locus. This may be achieved using 'bookmark' complementation-a simple CRISPR/Cas9-based strategy illustrated in Figure 1-relying on homology-directed repair (HDR) [11]. It is reliant on the prior incorporation into the mutant allele of a $24 \mathrm{nt}$ bookmark sequence between the start and stop codon of the gene to be deleted, which represents a single guide RNA (sgRNA) target for the Streptococcus pyogenes Cas9 nuclease (SpCas9). The bookmark comprises a 20 nt protospacer directly upstream of a $3 \mathrm{nt}$ protospacer adjacent motif (PAM) and is extended to 24 nt by the addition of a single random nucleotide before the protospacer or after the PAM. As this comprises eight codons, the replacement of the targeted gene by the mutant allele creates an in-frame deletion. The positioning of the single nucleotide either before the protospacer or after the PAM, and/or bookmark orientation relative to the start codon of the $\mathrm{KO}$ gene, can be varied to avoid premature stop codons.

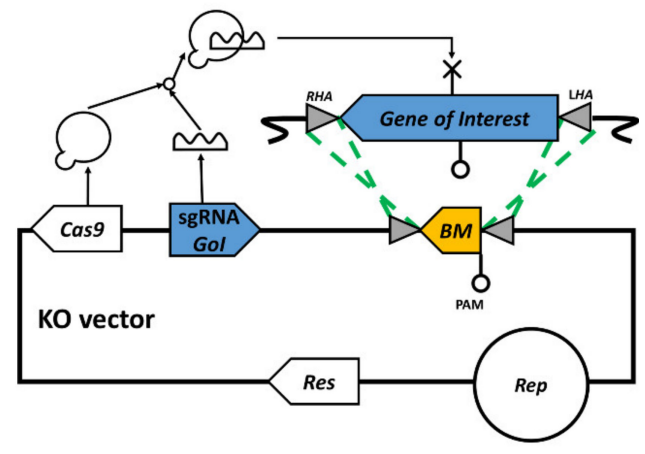

(a)

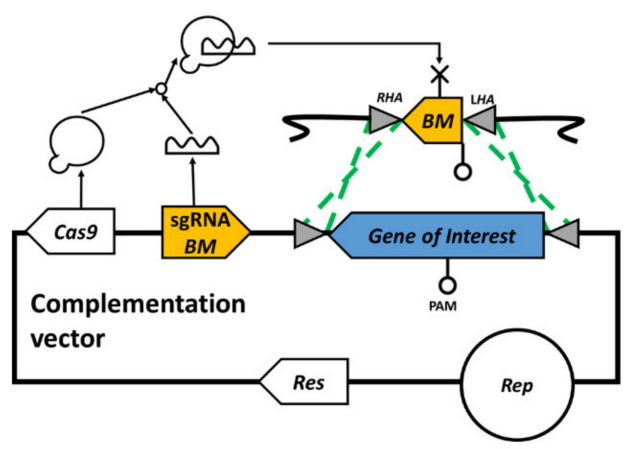

(b)

Figure 1. Overview of the bookmark complementation strategy. (a) First step-knockout of the gene of interest and insertion of the bookmark in its genomic locus using a knock-out vector consisting of a Cas 9 nuclease and single guide RNA (sgRNA) expression cassettes, as well as an editing template composed of one 24 nucleotides (nt) bookmark flanked by homology arms. (b) Second step-after isolation of the knockout $(\mathrm{KO})$ mutant and plasmid loss, another round of Cas9-mediated homology-directed mutagenesis is carried out with the help of a complementation vector, to restore the gene of interest in its original locus. The complementation vector is identical to the $\mathrm{KO}$ vector, except for its sgRNA cassette, which targets the genomic bookmark that was previously inserted, and editing template, which consists of the gene of interest flanked by the same homology arms. The gene of interest can be watermarked with a silent mutation for higher reliability of the complementation step. GoI: Gene of interest, Res: Antibiotic resistance marker, Rep: Replicon, BM: Bookmark, LHA: Left homology arm, RHA: Right homology arm.

When a typical CRISPR/Cas9 vector (incorporating a sgRNA that targets the bookmark) is used to replace the mutant allele with a WT copy of the gene by homologous recombination, the complemented strain generated is immune to the effects of Cas9. In contrast, the genome of the in-frame deletion mutant is cleaved due to the presence of the bookmark. This allows the rapid selection of complemented strains. The complemented strain obtained with the bookmark strategy is theoretically identical to the parental WT strain. As such, a silent mutation, or 'watermark', that can be detected by an appropriate polymerase chain reaction (PCR) screen should be inserted into the complementing gene [10]. Its presence eliminates any concerns that the experiment has been contaminated with the WT strain.

The validation of the principle of bookmark complementation was achieved in three steps. In the first instance, because the bookmark strategy is reliant on the sequence used being an effective target for SpCas9 and being absent in the organism being manipulated, we chose a total of nine different 
protospacers from three different bacterial species that had been successfully exploited in previous studies as SpCas9 targets. BLASTn (https://blast.ncbi.nlm.nih.gov) was then used to establish that the test organism to be manipulated here, $C$. autoethanogenum, did not contain equivalent sequences, and off-target efficiencies were calculated with the algorithm of Hsu et al. 2013 [12]. In the second step, the pyrE gene was replaced by a contiguous array of all nine bookmark protospacers and their respective PAM (BMa) using a previously described CRISPR/Cas9 editing system [6,7]. In the third and final step, nine CRISPR/Cas9 vectors incorporating a functional copy of pyrE and flanking homology arms, together with one of the nine sgRNAs each targeting a different bookmark protospacer, were used [6,7] to restore the inactivated genomic pyrE gene to WT. This particular gene was chosen because of the ease with which the mutant and WT allele can be phenotypically distinguished. The mutant allele confers uracil auxotrophy on the host as well as resistance to 5-fluoroorotic acid (5-FOA). WT strains are in contrast sensitive to 5-FOA and are uracil prototrophs.

\section{Materials and Methods}

\subsection{Strains and Medium}

Clostridium autoethanogenum strain DSM10061 was recovered from cryostocks and cultivated in pre-reduced yeast tryptone fructose (YTF) medium in an anaerobic cabinet (Don Whitley Scientific Ltd, Bingley, UK) at $37^{\circ} \mathrm{C}$ [13]. YTF solid medium was then complemented with the following antibiotics: $7.5 \mu \mathrm{g} / \mathrm{mL}$ thiamphenicol for transconjugants selection; $250 \mu \mathrm{g} / \mathrm{mL}$ D-cycloserine for elimination of the E. coli DNA donor strain; $1 \mathrm{mg} / \mathrm{mL}$ of 5 -fluoroorotic acid for $\triangle p y r E$ mutant selection. A $\triangle p y r E$ C. autoethanogenum strain was also generated to be used as a control for the colony PCR of the pyrE locus. This strain was generated through conjugation with pMTL431511_CLAU_pyrE [7],-—a standard pMTL vector [14] with a functional truncated Cas9, sgRNA targeting pyrE and editing template which leave only the first two and last two nucleotides of the pyrE gene in the pyrE locus after successful HDR.

Escherichia coli strain $\mathrm{K} 12 \mathrm{DH} 5 \alpha$ was used as host for vector assembly and cloning and was cultivated in Luria-Bertani (LB) broth with $12.5 \mu \mathrm{g} / \mathrm{mL}$ chloramphenicol for transformant selection. E. coli strain sExpress [15] was used as the conjugal DNA donor strain to transfer plasmids into C. autoethanogenum. It was grown on LB broth with $50 \mu \mathrm{g} / \mathrm{mL}$ kanamycin to maintain the vector R702, which enables conjugative transfer, with the addition of $12.5 \mu \mathrm{g} / \mathrm{mL}$ chloramphenicol to maintain the vectors to be conjugated into $C$. autoethanogenum.

\subsection{Basic Local Alignment Search Tool (BLAST) Search}

The parameters presented in Table 1 were used to BLAST all nine bookmark protospacers with their respective PAMs (23 bp) against the published Clostridium autoethanogenum genome (PubMed ID CP012395.1) [13].

Table 1. Parameters of the basic local alignment search tool (BLAST) of all the bookmark protospacers and their associated protospacer adjacent motif (PAM) against Clostridium autoethanogenum genomes.

\begin{tabular}{cc}
\hline Search Parameter & Value \\
\hline Program & BLASTn \\
Word size & 7 \\
Expect value & 1000 \\
Hitlist size & 100 \\
Match/Mismatch scores & $1,-1$ \\
Gapcosts & 5,2 \\
Filter string & $\mathrm{F}$ \\
Genetic Code & 1 \\
\hline Database & \\
\hline Posted date & Mar 24, 2020 9:05 AM \\
Number of letters & $8,771,469$ \\
Number of sequences & 18 \\
Entrez query & Includes: Clostridium autoethanogenum DSM 10061 (taxid:1341692) \\
\hline
\end{tabular}




\subsection{Off-Target Efficiency}

The parameters presented in Table 2 were used to calculate the off-target efficiency of each bookmark protospacer using the Molecular biology suite software suite of Benchling (San Francisco, CA, USA) and the algorithm of Hsu et al. [12].

Table 2. Parameters used to calculate the off-target efficiency (specificity score) of all nine bookmarks using the Molecular biology suite software suite of Benchling and the algorithm of Hsu et al. [12].

\begin{tabular}{cc}
\hline Parameter & Value \\
\hline Reference genome & GCA_000484505.1 (Clostridium autoethanogenum DSM 10061) \\
Design type & Single guide \\
Guide length & $20 \mathrm{bp}$ \\
PAM & NGG (SpCas9, 3'side) \\
\hline
\end{tabular}

\subsection{Vector Assembly}

All kits, enzymes, and buffers were purchased from New England Biolabs Ltd (Hitchin, UK) and used following the manufacturer's instructions unless specified. Primers and single stranded DNA (ssDNA) oligomers were ordered from Sigma-Aldrich Company Ltd (Gillingham, UK) and resuspended in $100 \mu \mathrm{M}$ solutions with TE buffer (10 mM Tris: $1 \mathrm{mM}$ EDTA; $\mathrm{pH}$ 8.0). Unless otherwise specified, all PCR reactions used Q5 high-fidelity polymerase $2 x$ master mix with whole $C$. autoethanogenum cells as DNA template and occurred after a 10 min denaturation at $98^{\circ} \mathrm{C}$, followed by 35 cycles of $10 \mathrm{~s}$ denaturation at $98^{\circ} \mathrm{C}, 15 \mathrm{~s}$ annealing at $60^{\circ} \mathrm{C}, 3 \mathrm{~min}$ elongation at $72^{\circ} \mathrm{C}$. Unless otherwise specified, vectors were assembled using HiFi assembly kit. In this case, all parts were designed to share between $25 \mathrm{nt}$ and $40 \mathrm{nt}$ of homologous sequences with their intended adjacent fragments. The sequence of all primers used in this study are available in Table S1.

The vector pMTL431511_CLAU_pyrE was digested with AsiSI and AscI, then dephosphorylated with Antarctic phosphatase and gel purified to remove its editing template. In parallel, the homology arms of the pyrE locus were amplified. The left homology arm was amplified using oFS109 with oFS119 and the right homology arm using oFS112 with oFS120. The amplicons were then run on a $1 \%(\mathrm{w} / \mathrm{v})$ agarose gel for gel extraction and elution in $6 \mu \mathrm{L}$ of de-ionised, nuclease free water. The Bookmark array was synthesised by Integrated DNA Technologies (IDT) Ltd (Sheffield, UK) and resuspended in $50 \mu \mathrm{L}$ de-ionized and nuclease-free $\mathrm{H}_{2} \mathrm{O}$. Finally, digested pMTL431511_CLAU_pyrE, left homology arm, right homology arm, and bookmark array were assembled together in one single step using a NEBuilder ${ }^{\circledR}$ HiFi DNA assembly cloning kit from New England Biolabs to form the vector pMTL431511_BMa.

The assembly of the bookmark complementation vectors was done in two steps. First, a new editing template meant to complement pyrE was introduced in the vector pMTL431511_CLAU_pyrE. The pyrE editing template was amplified using the PCR primers oFS66 and oFS67. After PCR clean-up, the pyrE editing template was digested with AsiSI and AscI and gel purified in $6 \mu \mathrm{L}$ elution buffer. Finally, $50 \mathrm{ng}$ of the digested and dephosporylated pMTL431511-CLAU-pyrE were ligated with $40 \mathrm{ng}$ of the digested pyrE editing template at $16^{\circ} \mathrm{C}$ overnight using T4 DNA ligase and associated buffer in a $20 \mu \mathrm{L}$ reaction, to form the intermediary pMTL431511_pyrE_sg3 vector.

In a second assembly step, the intermediary pMTL431511_pyrE_sg3 vector was digested with SalI and dephosphorylated with Antarctic phosphatase and gel purified in $6 \mu \mathrm{L}$ elution buffer. In parallel, stocks of double-stranded DNA (dsDNA) coding for the sgRNA targeting each bookmark were made by annealing together ssDNA oligomers. ssDNA oligomers annealing was achieved by mixing $5 \mu \mathrm{L}$ of $100 \mathrm{mM}$ solution of each oligomer (oFS39 to oFS47) with its respective reverse-complementary oligomer (oFS79 to oFS87), then incubating them $5 \mathrm{~min}$ at $98^{\circ} \mathrm{C}, 5 \mathrm{~min}$ at $72{ }^{\circ} \mathrm{C}$, and $5 \mathrm{~min}$ at $50{ }^{\circ} \mathrm{C}$ before holding the temperature at $15^{\circ} \mathrm{C}$. dsDNA sgRNA oligomers were then diluted 100-fold in New England Biolabs buffer 2.1 to form a dsDNA oligomer stock. Finally, the nine bookmark complementation vectors were 
assembled in parallel by HiFi assembly combining $1 \mu \mathrm{L}$ of their respective dsDNA bookmark oligomer stock with $50 \mathrm{ng}$ of the pMTL431511_pyrE_sg3 vector previously digested by SalI. These vectors were labelled pMTL431511_BM4 to pMTL431511_BM12 for their associated target bookmark protospacer.

\subsection{Cloning}

After vector assembly and transformation into $40 \mu \mathrm{L}$ of chemically competent $E$. coli $\mathrm{K} 12$ strain DH5 $\alpha$, single colonies were suspended in $40 \mu \mathrm{L}$ of sterile deionised water to be stored, used as DNA template for PCR screening, and used as inoculum for an overnight culture. Vectors were extracted from overnight cultures using a plasmid extraction kit and sent to Eurofins Genomics (Wolverhampton, UK) for Sanger sequencing.

\subsection{Conjugation into C. autoethanogenum}

Conjugative DNA transfer was undertaken following established methods $[7,15,16]$. Briefly, $1 \mathrm{~mL}$ of a E. coli sExpress strain carrying the target plasmid was grown to an OD600 of 0.4, centrifuged at $3000 \mathrm{G}$ for $3 \mathrm{~min}$, washed with $500 \mu \mathrm{L}$ phosphate-buffered saline solution (PBS), transferred in an anaerobic cabinet, and mixed with $0.2 \mathrm{~mL}$ of a $C$. autoethanogenum culture grown overnight to an OD600 of approximately 0.2. The cell mixture was spread on solid YTF without antibiotic and incubated for $24 \mathrm{~h}$. Afterward, cells were resuspended with $600 \mu \mathrm{L}$ PBS and spread onto YTF plates with $250 \mu \mathrm{g} / \mathrm{mL}$ D-cycloserine and $7.5 \mu \mathrm{g} / \mathrm{mL}$ thiamphenicol, respectively for counter-selection of the sExpress strain and selection of the $C$. autoethanogenum transconjugant. Single colonies were then screened by colony PCR.

\subsection{Colony Polymerase Chain Reaction (PCR)}

After conjugation of a vector into $C$. autoethanogenum, eight individual colonies were patched onto YTF selective medium to isolate mutants. After four days, the patches that grew were screened by PCR using the primers oFS105 and oFS106 and Q5 polymerase from New England Biolabs, along with a WT and a $\triangle p y r E$ strain of $C$. autoethanogenum as positive controls. Amplicons of the expected size were then purified using a QIAquick PCR Purification Kit from Qiagen (Manchester, UK) and validated by Sanger sequencing by Eurofins Genomics.

\subsection{Plasmid Loss and Cryopreservation}

Once a mutant strain had been confirmed by PCR, it was re-streaked on YTF plates without antibiotic to isolate single colonies. Single colonies were then re-streaked again, this time both on YTF medium with and without thiamphenicol selection. Colonies that failed to grow under thiamphenicol selection were further confirmed to have lost the vector by colony PCR with oFS68 and oFS75. Finally, the strain was inoculated into liquid YTF media and incubated for three days and $0.9 \mathrm{~mL}$ of culture used to generate cryostocks with $0.1 \mathrm{~mL}$ of dimethyl sulfoxide. These were then stored at $-80{ }^{\circ} \mathrm{C}$.

\section{Results}

\subsection{Bookmark Design}

Nine protospacer sequences were taken from successful examples of SpCas9 mutagenesis [17-19] and assigned an arbitrary PAM. Using BLAST to look for similar sequences in the genome of C. autoethanogenum (accession CP012395.1) highlighted no sequences identical to any of the $23 \mathrm{nt}$ bookmark candidates, File S1. The most similar hits were 19 non-consecutive identical bases out of 23 in BM9 (in position 223466) and 18 non-consecutive identical bases in BM11 (in position 3324583) and BM5 (in positions 1235296, 2165840 and 4340901). The off-target score of each bookmark protospacer was $>98 \%$, which confirmed them as suitable bookmark protospacers (Table S2). An extra nucleotide was then added to either extremity of each bookmark candidate to carry their length to $24 \mathrm{nt}$ and avoid the generation of an internal stop codon. The final bookmark sequences are summarised in Table 3. 
Table 3. Summary of the bookmark sequences. Nine protospacers were picked from the literature as successful examples of SpCas9 targets in bacterial species [17-19]. To constitute a bookmark, each protospacer should be immediately followed by a PAM (suggested: AGG) and complemented with one single nucleotide at either extremity. For each bookmark, the position of the extra nucleotide as well as the orientation of the bookmark relative to the coding sequence (CDS) it replaces are given to avoid internal STOP codons. "+": same direction as the target CDS, " - ": reverse-complementary direction relative to the target CDS, "+/" : either same or reverse-complementary direction relative to the target CDS, S. pneumonia: Streptococcus pneumonia, B. subtilis: Bacillus subtilis, L. reuteri = Lactobacillus reuteri.

\begin{tabular}{|c|c|c|c|c|c|c|}
\hline \multirow{2}{*}{ Bookmark } & \multicolumn{4}{|c|}{ Bookmark Sequence (24 nt) } & \multirow{2}{*}{ Orientation } & \multirow{2}{*}{ Origin } \\
\hline & Extra nt & Protospacer (20 nt) & PAM & Extra nt & & \\
\hline BM4 & G & AGGGTTGTGGGTTGTACGGA & AGG & / & $+/-$ & S. pneumonia \\
\hline BM5 & / & ATTTCTGATATTACTGTCAC & AGG & A & $+/-$ & S. pneumoniae \\
\hline BM6 & / & ACCGATACCGTTTACGAAAT & AGG & $\mathrm{A}$ & $+/-$ & S. pneumoniae \\
\hline BM7 & G & TGAAGATCAGGCTATCACTG & AGG & / & + & B. subtilis \\
\hline BM8 & $\mathrm{G}$ & TCCGGAGCTCCGATAAAAAA & TGG & / & $+/-$ & B. subtilis \\
\hline BM9 & G & TATTGATTCTCTTCAAGTAG & AGG & / & - & B. subtilis \\
\hline BM10 & / & CCATTGTACTATCATGCTAG & AGG & A & $+/-$ & L. reuteri \\
\hline BM11 & G & ATGCAGTCGGCTGTAGAAAG & AGG & / & $+/-$ & L. reuteri \\
\hline BM12 & G & CGACTGCATTTTATTATGTA & AGG & / & $+/-$ & L. reuteri \\
\hline
\end{tabular}

\section{2. pyrE Knock-Out and Bookmarks Knock-In}

The native pyrE gene of $C$. autoethanogenum was replaced with a mutant allele comprising the contiguous $207 \mathrm{nt}$ array of nine protospacer sequences and their respective PAM (Table S3), flanked by the first and last two codons of the pyrE gene. This was done using pMTL431511_BMa, a KO vector based on a previously described CRISPR/Cas9 vector which expresses a truncated Cas9 nuclease (trCas9) [7]. The editing template of pMTL431511_BMa comprises a left homology arm (LHA) and a right homology arm (RHA) that flank the protospacer array. The LHA essentially covers the $1 \mathrm{~kb}$ of DNA from the region upstream of pyrE but includes the first two codons of the gene. The RHA represents the $1 \mathrm{~kb}$ region downstream of $p y r E$, beginning with the last two codons of the gene. The sgRNA targets a region only present in the WT pyrE allele.

After conjugation of pMTL431511_BMa into C. autoethanogenum (Figure 2A, Figures S1 and S2), eight individual colonies were patched onto 5-FOA selective medium to isolate $\triangle p y r E:: B M a$ mutants. After four days, six patches had grown and were screened by colony PCR using the primers oFS105 and oFS106. Cells derived from WT and $\Delta p y r E$ strains of C. autoethanogenum were used as controls. Four colonies generated an amplified DNA fragment close to the predicted size of $1.7 \mathrm{~kb}$ (Figure 2B), albeit weakly in the case of lanes 3 and 4 . Sanger sequencing of the amplified DNA fragment of a randomly selected clone (lane 1) confirmed the replacement of pyrE with the bookmark array. The chosen strain was re-streaked on plates without antibiotic to lose the $\mathrm{KO}$ vector before storage in cryostocks. 


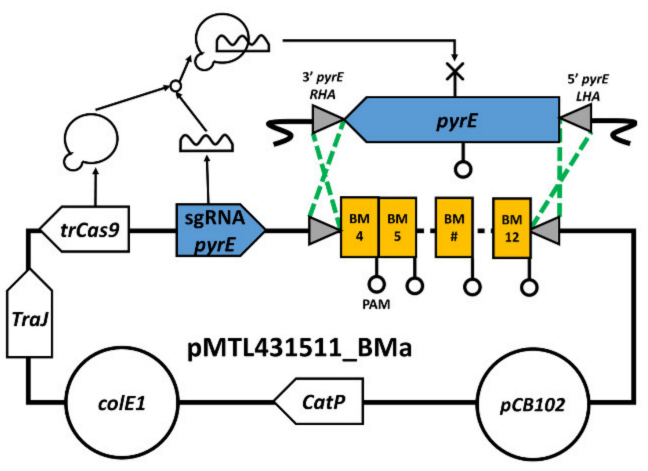

(a)

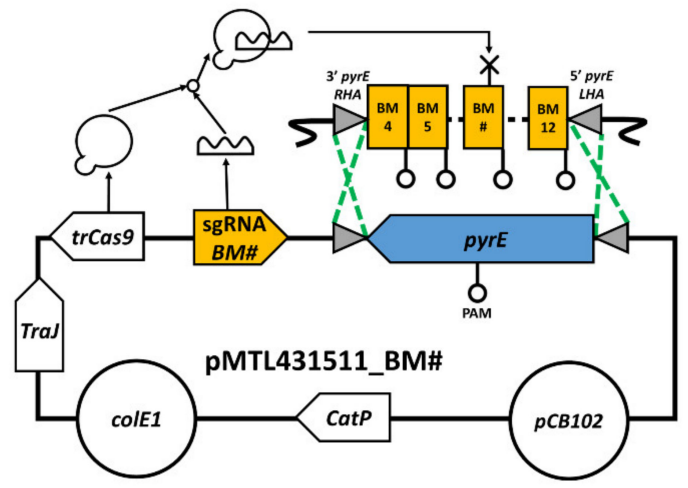

(c)

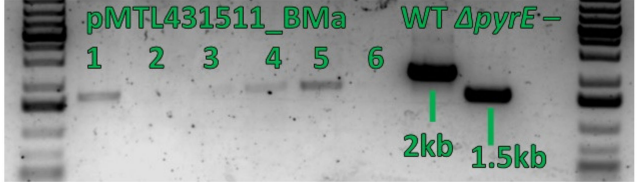

(b)

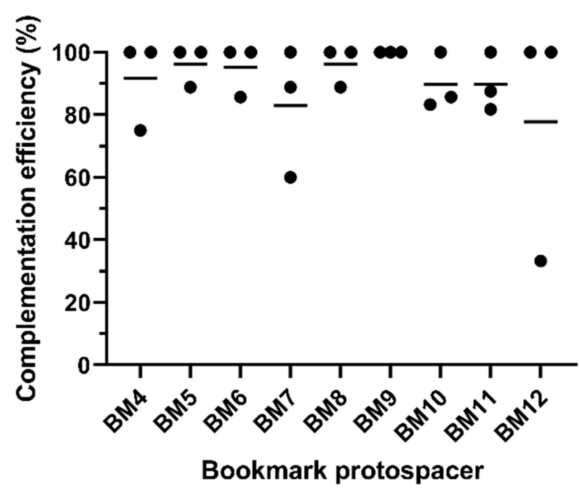

(d)

Figure 2. Proof of principle of the bookmark strategy. (a) Integration of an array of bookmark protospacers into the genomic locus of pyrE using pMTL431511_BMa. The bookmark protospacer array (BM4 to BM12) is flanked by two homology arms of $1 \mathrm{~kb}$, each homologous to the genomic region directly upstream (LHA) and downstream (RHA) of the pyrE locus and which include the first two and last two codons of pyrE. Homology directed mutagenesis of the pyrE locus replaces the pyrE gene with the protospacer array. trCas9 nuclease and a sgRNA expression cassettes counter-select wild type (WT) transconjugants by cutting the genomic DNA of cells which have a WT pyrE locus. (b) Electrophoresis gel of six C. autoethanogenum colonies obtained after conjugation of pMTL431511_BMa. The pyrE locus of each colony was amplified using the primers oFS105 and oFS106 then run on a 1\% (w/v) agarose gel. The expected size of the amplicon of a successfully knocked-in bookmark array (BMa) is $1.7 \mathrm{~kb}$, versus $2 \mathrm{~kb}$ for the WT pyrE locus and $1.5 \mathrm{~kb}$ for a clean $\triangle p y r E$ genotype without the bookmark array. No DNA template was added to the PCR mix of the negative control (-). (c) Integration of the pyrE gene back in its original locus using pMTL431511_BM\# to target different bookmark protospacers. Each homology arm consists of a $1 \mathrm{~kb}$ region directly upstream (LHA) or downstream (RHA) of the pyrE locus, including the first two and the last two nucleotides of the pyrE gene, respectively. Together with the pyrE gene they constitute the pyrE editing template. trCas9 nuclease and sgRNA expression counter-selects pyrE $\triangle:: B M a$ transconjugants by cutting the genomic DNA of cells with an intact bookmark protospacer array. (d) Complementation efficiency of nine protospacers in C. autoethanogenum. Complementation of a pyrE $\triangle:: B M a$ strain of $C$. autoethanogenum using different bookmark protospacers was successful in $91 \pm 15 \%$ of all screened colonies, with little to no variations observed in between the particular heterologous protospacers targeted by each bookmark complementation vectors. $(\bullet)$ : complementation efficiency of one separate conjugation, (-): arithmetic mean of the complementation efficiency of three independent conjugations for each bookmark, TraJ: conjugative transfer function, colE1: Gram-negative replicon, CatP: chloramphenicol/thiamphenicol resistance cassette, $p$ CB102: Gram-positive repliconBM\#: Protospacer sequence of any bookmark, where \# is an integer between four and 12.

\subsection{Bookmark Complementation of pyrE}

Having generated a $\mathrm{KO}$ strain carrying a pyrE mutant allele, it was restored to WT using CRISPR/trCas9 vectors (pMTL431511_BM4 to pMTL431511_BM12) which harboured one of nine 
sgRNA cassettes targeting each of the bookmark sequences (Table S4) and a $2.5 \mathrm{~kb}$ region encompassing the entire pyrE gene as editing template (Figure 2C). Following transfer of these plasmids into the $\triangle p y r E:: B M a$ strain, colony PCR was undertaken on the transconjugants obtained to ascertain whether they carried the WT or mutant allele (Figures S3-S11). To calculate the complementation efficiency of each bookmark protospacer, the number of colonies that reverted to WT after conjugation was divided by the total number of colonies screened (Table S5). Each complementation was independently performed three times (Figure 2D).

The average complementation efficiency was $91 \pm 15 \%$, with BM9 having consistently the maximal efficiency (out of seven, seven, and five colonies screened for each replicate respectively). The lowest efficiency was achieved by BM12, with one of its three replicates achieving only $33 \%$ efficiency (only two out of six colonies were successfully complemented); however, the other two replicates of BM12 achieved 100\% efficiency (four out of four colonies were complemented in both cases). Taken together, these results show that all bookmark complementation vectors are probably equivalent in their ability to target the protospacers used in each genomic bookmark and to replace them by a gene such as pyrE.

\section{Discussion}

The combined data obtained established the proof of principle for a new gold standard complementation strategy that is broadly applicable to any organism compatible with CRISPR/ Cas9-mediated homology-directed mutagenesis. By complementing the gene of interest back into its original locus, bookmark complementation avoids the biases of traditional complementation methods such as an extra metabolic burden or the disruption of an additional genomic locus. Additionally, the bookmarks are designed to produce in-frame mutations without internal stop codons so as to minimise the chances of inducing polar effects. Here, we describe and characterise a small library of nine functional protospacers that were highly effective as bookmarks for complementation in C. autoethanogenum. However, they would be equally applicable to any organism which lack homologous sequences in their genome.

It is clear that bookmark technology will prove useful beyond complementation, such as during the sequential assembly of large genomic operons or to assist various genome editing strategies [20].

Supplementary Materials: The following are available online at http://www.mdpi.com/2073-4425/11/4/458/s1, Table S1: Primers summary, Table S2: Off-target efficiency (specificity score) of all nine bookmark protospacers, Table S3: DNA synthesis summary, Table S4: Seed sequences used in sgRNA, Table S5: Raw data from the calculation of the complementation efficiency of each bookmark protospacer, Figure S1: Uncropped electrophoresis gel of six C. autoethanogenum colonies obtained after conjugation of pMTL431511_BMa, Figure S2: Subsequent PCRs of the $\triangle p y r E:: B M a$ strain screened in Figure S1, Figures S3-S11: Electrophoresis gel of Clostridium autoethanogenum $\triangle$ pyrE::BM4-12 colonies obtained after conjugation of pMTL431511_BM4 to BM12, File S1: BLAST of BM4-12.txt.

Author Contributions: Conceptualization, P.R.; methodology, P.R., C.M.H., and N.P.M.; investigation, F.M.S.; writing-original draft preparation, F.M.S.; writing—review and editing, C.M.H., E.L.B., and N.P.M. All authors have read and agreed to the published version of the manuscript.

Funding: This research was funded by the Biotechnology and Biological Sciences Research Council (grant numbers BB/L013940/1, BB/K00283X/1 and BB/L502030/1).

Acknowledgments: F.M.S. acknowledges the receipt of a studentship from the University of Nottingham, Synthetic Biology Research Centre (SBRC) Doctoral Training Programme.

Conflicts of Interest: The authors declare no conflict of interest. The funders had no role in the design of the study; in the collection, analyses, or interpretation of data; in the writing of the manuscript, or in the decision to publish the results.

\section{References}

1. Smits, W.K.; Lyras, D.; Lacy, D.B.; Wilcox, M.H.; Kuijper, E.J. Clostridium difficile infection. Nat. Rev. Dis. Prim. 2016, 2, 16021. [CrossRef] [PubMed]

2. Peck, M. Bacteria: Clostridium botulinum. In Encyclopedia of Food Safety; Motarjemi, Y., Ed.; Academic Press: Waltham, MA, USA, 2014; Volume 1, pp. 381-394. [CrossRef] 
3. Dürre, P. Fermentative production of butanol—the academic perspective. Curr. Opin. Biotechnol. 2011, 22, 331-336. [CrossRef] [PubMed]

4. Bengelsdorf, F.R.; Straub, M.; Dürre, P. Bacterial synthesis gas (syngas) fermentation. Environ. Technol. 2013, 34, 1639-1651. [CrossRef] [PubMed]

5. Cañadas, I.C.; Groothuis, D.; Zygouropoulou, M.; Rodrigues, R.; Minton, N.P. RiboCas: A universal CRISPR-Based editing tool for Clostridium. ACS Synth. Boil. 2019, 8, 1379-1390. [CrossRef] [PubMed]

6. Huang, H.; Chai, C.; Li, N.; Rowe, P.; Minton, N.P.; Yang, S.; Jiang, W.; Gu, Y. CRISPR/Cas9-based efficient genome editing in Clostridium ljungdahlii, an autotrophic gas-fermenting bacterium. ACS Synth. Boil. 2016, 5, 1355-1361. [CrossRef] [PubMed]

7. Ingle, P.; Groothuis, D.; Rowe, P.; Huang, H.; Cockayne, A.; Kuehne, S.A.; Jiang, W.; Gu, Y.; Humphreys, C.M.; Minton, N.P. Generation of a fully erythromycin-sensitive strain of Clostridioides difficile using a novel CRISPR-Cas9 genome editing system. Sci. Rep. 2019, 9, 8123. [CrossRef] [PubMed]

8. Minton, N.P.; Ehsaan, M.; Humphreys, C.M.; Little, G.; Baker, J.; Henstra, A.M.; Liew, F.; Kelly, M.L.; Sheng, L.; Schwarz, K.; et al. A roadmap for gene system development in Clostridium. Anaerobe 2016, 41, 104-112. [CrossRef] [PubMed]

9. Ullmann, A.; Jacob, F.; Monod, J. Characterization by in vitro complementation of a peptide corresponding to an operator-proximal segment of the $\beta$-galactosidase structural gene of Escherichia coli. J. Mol. Boil. 1967, 24, 339-343. [CrossRef]

10. Ng, Y.K.; Ehsaan, M.; Philip, S.; Collery, M.M.; Janoir, C.; Collignon, A.; Cartman, S.T.; Minton, N.P. Expanding the repertoire of gene tools for precise manipulation of the Clostridium difficile Genome: Allelic exchange using pyrE alleles. PLoS ONE 2013, 8, e56051. [CrossRef] [PubMed]

11. Mougiakos, I.; Bosma, E.F.; De Vos, W.M.; Van Kranenburg, R.; Van Der Oost, J. Next generation prokaryotic engineering: The CRISPR-Cas toolkit. Trends Biotechnol. 2016, 34, 575-587. [CrossRef] [PubMed]

12. Hsu, P.; A Scott, D.; A Weinstein, J.; Ran, F.A.; Konermann, S.; Agarwala, V.; Li, Y.; Fine, E.; Wu, X.; Shalem, O.; et al. DNA targeting specificity of RNA-guided Cas9 nucleases. Nat. Biotechnol. 2013, 31, 827-832. [CrossRef] [PubMed]

13. Humphreys, C.M.; McLean, S.; Schatschneider, S.; Millat, T.; Henstra, A.M.; Annan, F.J.; Breitkopf, R.; Pander, B.; Piatek, P.; Rowe, P.; et al. Whole genome sequence and manual annotation of Clostridium autoethanogenum, an industrially relevant bacterium. BMC Genom. 2015, 16, 1085. [CrossRef] [PubMed]

14. Heap, J.T.; Pennington, O.J.; Cartman, S.T.; Minton, N.P. A modular system for Clostridium shuttle plasmids. J. Microbiol. Methods 2009, 78, 79-85. [CrossRef] [PubMed]

15. Woods, C.; Humphreys, C.M.; Rodrigues, R.M.; Ingle, P.; Rowe, P.; Henstra, A.M.; Köpke, M.; Simpson, S.D.; Winzer, K.; Minton, N.P. A novel conjugal donor strain for improved DNA transfer into Clostridium spp. Anaerobe 2019, 59, 184-191. [CrossRef] [PubMed]

16. Purdy, D.; O’Keeffe, T.A.T.; Elmore, M.; Herbert, M.; McLeod, A.; Bokori-Brown, M.; Ostrowski, A.; Minton, N.P. Conjugative transfer of clostridial shuttle vectors from Escherichia coli to Clostridium difficile through circumvention of the restriction barrier. Mol. Microbiol. 2002, 46, 439-452. [CrossRef] [PubMed]

17. Jiang, W.; Bikard, D.; Cox, D.; Zhang, F.; A Marraffini, L. RNA-guided editing of bacterial genomes using CRISPR-Cas systems. Nat. Biotechnol. 2013, 31, 233-239. [CrossRef] [PubMed]

18. Altenbuchner, J. Editing of the Bacillus subtilis genome by the CRISPR-Cas9 system. Appl. Environ. Microbiol. 2016, 82, 5421-5427. [CrossRef] [PubMed]

19. Oh, J.-H.; Van Pijkeren, J.-P. CRISPR-Cas9-assisted recombineering in Lactobacillus reuteri. Nucleic Acids Res. 2014, 42, e131. [CrossRef] [PubMed]

20. Finnigan, G.C.; Thorner, J. mCAL: A new approach for versatile multiplex action of Cas9 using one sgRNA and loci flanked by a programmed target sequence. Genes Genomes Genet. 2016, 6, 2147-2156. [CrossRef] [PubMed]

(C) 2020 by the authors. Licensee MDPI, Basel, Switzerland. This article is an open access article distributed under the terms and conditions of the Creative Commons Attribution (CC BY) license (http://creativecommons.org/licenses/by/4.0/). 